\title{
Agôn
}

Revue des arts de la scène

$4 \mid 2011$

L'objet

\section{Un arrangement d'objets et de personnes : étude de Parts of Some Sextets d'Yvonne Rainer}

Johanna Renard.

\section{(2) OpenEdition}

Journals

Édition électronique

URL : http://journals.openedition.org/agon/1988

DOI : 10.4000/agon.1988

ISSN : 1961-8581

Éditeur

Association Agôn

Référence électronique

Johanna Renard., "Un arrangement d'objets et de personnes : étude de Parts of Some Sextets d'Yvonne Rainer », Agôn [En ligne], 4 | 2011, mis en ligne le 19 décembre 2011, consulté le 17 avril 2020. URL : http://journals.openedition.org/agon/1988; DOI : https://doi.org/10.4000/agon.1988 


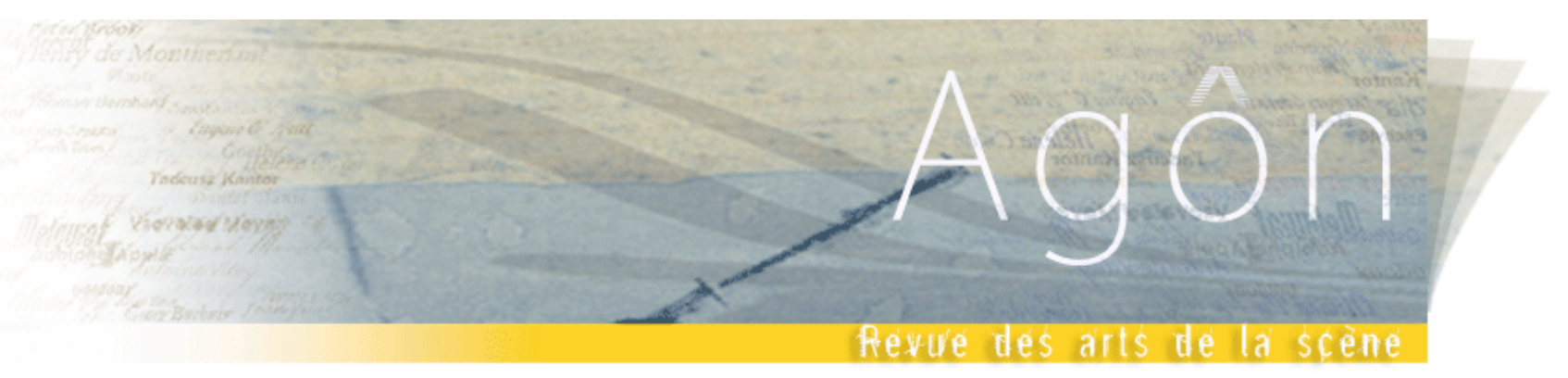

Un arrangement d'objets et de personnes: étude de Parts of Some Sextets d'Yvonne Rainer

Johanna Renard

\begin{abstract}
Résumé
Parts of Some Sextets (1965), une « danse pour dix personnes et douze matelas », est une pièce charnière dans la carrière de la chorégraphe américaine Yvonne Rainer, qui l'entraîna à théoriser sa conception de la danse. Affirmant que les objets et les corps peuvent être interchangeables, elle utilise les corps comme des «agents neutres " afin de se débarrasser du narcissisme et du culte de la personnalité dont elle dénonce la prééminence en danse. Ainsi, Parts of Some Sextets se compose d'actions inspirées des mouvements les plus anodins de la vie quotidienne. Surtout, Rainer offre le rôle principal au matelas, objet à la fois atypique et absurde, avec lesquels les danseurs doivent interagir. En analysant la place centrale offerte à l'objet matelas dans cette chorégraphie, on s'attachera à mettre en lumière comment la remise en question du traitement de l'objet a permis à l'artiste de profondément repenser sa pratique. Pour ce faire, on mettra en évidence l'influence cruciale que la conception de l'objet développée dans la théorie du nouveau roman d'Alain Robbe-Grillet a exercée sur Yvonne Rainer.
\end{abstract}

MATELAS [mat([?])la] n.m. Pièce de literie, long et large coussin rembourré que l'on étend d'ordinaire sur le sommier d'un lit. ${ }^{1}$

Objet encombrant, incommode, volumineux, voire même pataud, balourd, gauche, le matelas semble bien éloigné de l'univers de la danse, presque antinomique - ou, plus exactement, ce sont les significations dont il est investi lorsqu'on l'appréhende selon notre système de références habituel. Du fait de son inertie, il freine toute aspiration au mouvement, à la légèreté, à l'agilité, à la souplesse et, par-dessus tout, à la grâce, traditionnellement et sempiternellement associée au geste dansé. Pourtant, il n'en demeure pas moins l'un des objets les plus récurrents et les plus essentiels dans l'œuvre de la chorégraphe américaine, Yvonne Rainer. Il est ainsi la vedette de l'une de ses pièces les plus radicales, Parts of Some Sextets (1965), qui renouvelle fondamentalement la place de l'objet en danse. En effet, cette dernière donne à voir aux spectateurs la pure physicalité des corps ${ }^{2}$ et la matérialité des choses, jusque là escamotée dans la danse moderne, qui met l'accent sur l'expressivité dramatique. Marquée par les recherches de la chorégraphe californienne Anna Halprin sur le geste quotidien, cette chorégraphie est conçue comme «[the] arrangement of materials and people ${ }^{3}$ » (l'arrangement de matériaux et de personnes). Qu'implique cette réassignation des rôles ? Dans quelle mesure ce nouveau rapport au monde matériel permet-il de réformer et de régénérer l'art chorégraphique ? À travers l'exploration de la place de l'objet matelas dans Parts of Some Sextets ${ }^{4}$, on s'attachera à démontrer

${ }^{1}$ Le Nouveau Petit Robert, Paris, 2008, p.1550.

${ }^{2}$ Le terme "physicalité » est ici employé dans le sens anglo-saxon, en traduction de "physicality », défini par l'Oxford English Dictionary comme «le fait, la qualité ou la condition d'être physique ». Oxford English Dictionary, troisième édition, mars 2006, http://www.oed.com.rproxy.sc.univ-paris-diderot.fr/view/Entry/143124, [site consulté le 19 November 2011].

${ }^{3}$ Yvonne Rainer, Work, 1961-1973, Halifax, The Press of Novia Scotia College of Art and Design, 1974, p. 47. Toutes les traductions de l'article ont été réalisées par l'auteur.

${ }^{4}$ Dans la suite du texte, le titre de cette œuvre sera abrégé en PoSS. 
comment l'utilisation de l'objet en danse a pu influencer de manière décisive à la fois la théorie et la pratique de l'artiste. Après avoir examiné la genèse de cette pièce, on abordera l'influence exercée par le nouveau roman, et plus particulièrement par son théoricien Alain Robbe-Grillet, sur la conception de l'objet développée par Rainer. Enfin, on mettra en évidence que la redéfinition de la place de l'objet remet profondément en cause l'humanisme et l'anthropocentrisme qui étaient la règle jusque là en danse.

\section{Danse avec les matelas}

En tant que danseuse et chorégraphe, Yvonne Rainer a considérablement marqué l'histoire de l'avant-garde new-yorkaise. Durant les années 1960, sa recherche chorégraphique s'épanouit au sein du Judson Dance Theater, qu'elle contribue à fonder en 1962, et entretient un dialogue fécond avec la scène artistique new-yorkaise, qui se concentre alors à New York Downtown. Rejetant tout autant le vocabulaire technique et esthétique extrêmement codifié imposé par le ballet que l'expressivité lyrique et la psychologisation perpétuées par la danse moderne, le Judson Dance Theater développe une danse dé-théâtralisée, souvent minimaliste, recourant à des procédés aussi divers que l'usage de méthodes de composition aléatoires, le collage, la répétition, l'improvisation, et offrant au corps, dans sa réalité la plus physique et la plus concrète, une place centrale. Dans ce cadre, le travail de Rainer est marqué par un rejet de la narration, des costumes et des décors, mais aussi de tout ce qui tend à instaurer une relation de fascination avec le spectateur. Concevant la création chorégraphique comme un processus en constante évolution, elle déconstruit les notions d'emphase, d'accentuation et d'intensité en danse, notamment dans le fameux Trio $A$ (1966), qui a consacré sa reconnaissance. Ses chorégraphies sont structurées comme des jeux, limités par quelques règles, et se développent autour de l'exécution de tâches inspirées de la vie quotidienne. En effet, Rainer fut fortement influencée par un bref séjour durant l'été 1960 au sein du Dancers's Workshop, créé par Anna Halprin dans les environs de San Francisco. Ayant largement contribué à fonder la danse postmoderne, Halprin préconise un retour $\mathrm{au}$ corps «objectif» et une intégration des mouvements de la vie quotidienne en danse. Recherchant des alternatives aux notions de style et de technique, elle fonde sa pratique sur des actions simples, qu'elle appelle les «tasks » (tâches), balayer le sol, verser de l'eau, collecter des végétaux, etc. Ce faisant, elle propose de revenir à une qualité essentielle du geste, débarrassé des artifices de la virtuosité et de la grâce. De la même manière, la danse de Rainer s'inspire des actions les plus banales (marcher, courir, ramper, se coucher, etc.), donnant la primauté au «factuel» et au concret. De ce fait, on ne s'étonnera guère que les objets les plus courants et anodins aient une place aussi prépondérante dans le travail de Rainer. Oreillers, tapis en caoutchouc, cordes, ballons, valises, reviennent de manière récurrente, telle une troupe inanimée avec laquelle les danseurs en chair et en os doivent interagir. Le matelas devient un objet d'inspiration chorégraphique pour Rainer en 1964, lors d'une représentation de la pièce Room Service à Philadelphie. Peu connue et peu documentée, cette dernière chorégraphie est cependant une pièce significative dans la carrière d'Yvonne Rainer. Ambitieuse et tentaculaire, elle met en scène trois équipes de danseurs se mouvant selon des règles de jeux rigoureusement prédéfinies au milieu de constructions très élaborées conçues par le sculpteur Charles Ross et constamment manipulées, agencées et réagencées par ce dernier et deux assistants. C'est lors de cette représentation que la chorégraphe découvrit le champ de possibilités offert par la manipulation d'un simple matelas :

Deux d'entre nous transportent un matelas, à travers la sortie arrière, jusqu'au couloir, puis font le tour pour revenir par une entrée latérale. En trimballant cet objet encombrant, en le retirant puis en le réintroduisant sur scène, quelque chose d'à la fois ridicule et de réjouissant émerge. II n'y a besoin d'aucune stylisation. Cette action 
semble tellement autonome qu'elle ne nécessite aucun fardage artistique, aucune justification ${ }^{5}$.

Rainer est séduite par le côté extrêmement prosaïque de cet objet sans distinction, dépourvu de beauté, d'expressivité, de magie, et qui se résume à sa fonction pratique. Or, à ce moment précis de sa carrière, la chorégraphe souhaite abandonner le style de ses premières pièces, notamment Three Satie Spoons (1961), Ordinary Dance ou Three Seascapes (1962), marquées par une certaine fougue extravagante :

Il devenait impératif de trouver une nouvelle façon de bouger. Je sentais que je ne pouvais plus faire appel à l'énergie, aux attaques violentes et impulsives qui avaient auparavant caractérisé mon travail. Je ne voulais plus non plus persister dans l'exploration de ce type de mouvements excentriques inspirés de la vie quotidienne que quelqu'un avait un jour décrit comme du « glamour loufoque» 6 .

C'est avec cet objectif en tête et en se saisissant du puissant potentiel du matelas, révélé par Room Service, qu'elle entreprend l'élaboration de Parts of Some Sextets. Créée pour dix personnes et douze matelas à partir d'une charte rigoureuse, cette pièce fut présentée au Wadsworth Atheneum à Hartford dans le Connecticut et à la Judson Memorial Church à New York en mars 1965. Elle rassemblait des danseurs professionnels ou occasionnels (parmi lesquels Steve Paxton, Robert Morris, Lucinda Childs et Robert Rauschenberg) et se composait de trente-et-une activités différentes, détaillées dans la charte qui structure la pièce : des séquences de marche ou de course, des manipulations de matelas, des moments d'immobilité, etc. Ces actions, inspirées de la vie quotidienne, se déroulaient isolément ou simultanément durant quarante-trois minutes, selon un timing rigoureux - un changement brusque d'activité intervenant toutes les trente secondes. Les changements d'activités se déroulaient en fonction de "signaux » prédéterminés présents dans l'enregistrement sonore accompagnant la pièce. Ce dernier était composé d'extraits du journal intime du Révérend William Bentley, lus par Yvonne Rainer et offrant un exposé à la fois austère et détaillé des faits du quotidien7. Précisons aussi qu'aux mouvements relevant davantage de la notion de «task » halprinienne, s'ajoutaient des sections plus ordonnancées, paraissant être des séquences conçues à partir de phrases consécutives. Par exemple, "Corridor Solo », l'une des différentes séries d'actions possibles, est peut-être moins éloigné de la conception traditionnelle de la séquence chorégraphiée : ainsi, il s'agit d'effectuer des mouvements d'échauffement très lentement et sans leur insuffler d'énergie. Enfin, il faut compléter cette présentation de la pièce en ajoutant que les différentes activités se déroulaient toutes en même temps sur scène. En ce sens, Rainer offre une visibilité totale aux spectateurs, lui assurant de ne rien perdre des actions performées ${ }^{8}$.

S'il est difficile de se faire une idée précise et juste d'une danse dont il ne reste que quelques photographies et des textes ${ }^{9}$, il n'en est pas moins certain que PoSS concentre les

5 Yvonne Rainer, "Some Retrospective Notes on a Dance for 10 People and 12 Mattresses Called Parts of Some Sextets", The Tulane Drama Review, vol. 10, n² 2, hiver 1965, p. 168. Désormais, les renvois à cet article seront signalés par la mention $S R$, suivie du numéro de page. Cette traduction et les suivantes ont été effectuées par l'auteur.

${ }^{6}$ Yvonne Rainer, SR, p. 170.

7 Voir William Bentley, The diary of William Bentley, D.D., pastor of the East Church, Salem, Massachusetts, Gloucester Mass., P. Smith, 1962. Durant près d'une trentaine d'années ce pasteur protestant rendit consciencieusement compte des événements de la vie quotidienne à Salem, Massachussetts (naissances, décès, mariages) ou de faits plus anecdotiques (ses amitiés et ses inimitiés, ses finances, etc.). Il faut noter que durant l'automne 1964, sa pièce déjà en gestation, Yvonne Rainer passa cinq semaines à lire et copier des extraits de ce journal à la New York Public Library.

8 Dans l'ouvrage Danse(s) performative(s), Céline Roux évoque les interactions entre danse et performance. Selon elle, si les deux pratiques renvoient à des acceptions diverses, elles se rejoignent durant la seconde moitié du XXe siècle au sein de la danse postmoderne notamment, lorsque les danseurs développent une réflexion autour de la représentation et du spectacle et en rejettent les normes conventionnelles. Ils mettent alors en place un nouveau rapport avec le public, chorégraphient des actions plus que des mouvements dansés traditionnels, s'inspirent du quotidien, investissent de nouveaux espaces, etc., élaborant ainsi une «attitude performative». Afin de mettre en évidence la porosité entre danse et performance durant cette période, nous reprendrons dans cet article le vocabulaire utilisé par Rainer elle-même et nous utiliserons donc tout autant des termes empruntés à la danse qu'à la performance. Voir Céline Roux, Danse(s) performative(s), Paris, L'Harmattan, 2007.

${ }^{9}$ La documentation de PoSS a été publiée dans Yvonne Rainer, Work, op. cit., pp. 44-61. 
innovations les plus marquantes d'Yvonne Rainer - et ce même si cette pièce est bien moins connue que le fameux Trio A. Plus important encore, PoSS peut être considérée comme le moment charnière dans sa carrière qui l'entraina à théoriser sa conception de la danse. Ainsi, c'est très significativement en conclusion d'un article relatant le processus de création à l'origine de PoSS qu'Yvonne Rainer a forgé son fameux «No Manifesto», texte lapidaire énumérant tous les oripeaux dont la danse doit, selon elle, se débarrasser :

«NON au grand spectacle non à la virtuosité non aux transformations et à la magie et au faire-semblant, non au glamour et à la transcendance de l'image de la vedette non à l'héroïque non à l'anti-héroïque non à la camelote visuelle non à l'implication de l'exécutant ou du spectateur non au style non au kitsch non à la séduction du spectateur par les ruses du danseur non à l'excentricité non au fait d'émouvoir ou d'être ému. ${ }^{10}$ »

Adoptant une position antagoniste, elle rejette de manière particulièrement radicale tout artifice en danse. Ce rejet se manifeste par un retour à la réalité tangible, concrète, et, de ce fait, à des mouvements ordinaires ne requérant pas de virtuosité particulière pour être exécutés. En ce sens, il nous semble que l'importance croissante de l'objet dans le travail de Rainer à partir de PoSS constitue un des pivots centraux de la nouvelle direction que la chorégraphe insuffle à la danse. Désormais, afin de traduire la pure matérialité des corps, en tant que réalités tangibles, il faut s'inspirer des propriétés de l'objet, cette réalité statique et non individuée simplement définie par sa matière et sa destination. Le mouvement lui-même doit être réduit à l'état d'objet: dynamique, rythme, emphase et tension doivent être abandonnés. Il ne s'agit plus que d'exécuter le geste de manière plate, neutre - au sens de détachée, impersonnelle, sans relief et uniforme - et dans le temps qu'il faudrait réellement pour le réaliser dans la vie ordinaire. L'usage du matelas et de ses propriétés physiques enrayent toute tentante envolée, toute facile séduction. Surtout, Rainer initie une réflexion sur l'interaction qui peut être créée entre le corps du danseur et l'objet. Elle s'intéresse ainsi aux relations externes que le corps ou l'objet entretient avec son environnement immédiat et aux mouvements qu'il peut subir ou causer. Citons en exemple l'une des activités de PoSS, dénommée "Human fly », qui demande aux danseurs de se jeter, le corps à l'horizontale et les bras en avant, sur une pile de matelas. Dans cette confrontation plutôt violente avec l'objet, le geste dansé est généré par le choc que la danseuse ou le danseur impose au matelas. Cependant, en heurtant le matelas et en rebondissant à sa rencontre, le corps est dans l'impossibilité de contrôler son mouvement. En ce sens, le matelas, ou plus exactement les matelas, influent de manière non négligeable sur la qualité du mouvement au moment de la réunion des deux corps (la matière et le corps vivant). Ainsi, même si les spectateurs perçoivent le matelas en tant que matière inerte qui ne paraît pas être en capacité de se mouvoir d'elle-même, au moment du choc, le mouvement communiqué produit un impact tout autant sur le corps que sur l'objet. Rainer est intéressée par les effets produits par cette collision sur le mouvement qui lui permettent d'insister sur le caractère purement matériel du corps ${ }^{11}$. Corps et objets deviennent autant d'outils dont elle met en exergue les qualités respectives en termes de masse, de poids ou de taille. C'est un univers entièrement objectivé, modelé sur la réalité extérieure de l'objet, que la chorégraphe met en scène. Afin de tendre à la plus grande neutralité possible, à une quasiindifférence, il est conçu indépendamment du sujet pensant. En définitive, c'est un nouveau regard sur le monde qui est proposé au spectateur et, même si cela peut sembler être un lieu commun, inlassablement ressassé lorsque l'on évoque la danse de Rainer ${ }^{12}$, il est somme toute pertinent de parler de danse « objective ».

10 Yvonne Rainer, SR, p. 178.

11 Il est intéressant de rappeler ici que Rainer ira plus loin dans sa remise en cause du dualisme classique dans sa pièce The Mind is a Muscle (1966-1968), qui refuse de souscrire à la distinction radicale entre la subjectivité et le monde objectif.

12 Voir notamment Barbara Rose, “ABC Art”, Art in America, vol. 53, n5, octobre 1965, pp. 55-72 ou Sally Banes, Terspichore in Sneakers, [1980], Middletown, CT, Wesleyan University Press, 1987. 


\section{L’influence de la littérature « objective » d'Alain Robbe-Grillet}

L'existence propre, indépendante et autonome conférée par Rainer aux matelas dans PoSS tout comme sa vive prédilection pour la réalité la plus tangible et concrète nous semble résulter de l'influence du nouveau roman, et plus particulièrement de son principal théoricien Alain RobbeGrillet. En effet, Rainer a décrit comme une véritable révélation la lecture de l'essai Pour un nouveau roman $(1963)^{13}$. Il est aisé de mettre en lumière les nombreuses correspondances entre les idées défendues par l'écrivain et la quête artistique de la chorégraphe. Ainsi, tous deux prônent la mort du personnage et la disparition de l'intrigue, l'usage d'un style descriptif ou le choix d'un formalisme radical. Mais Rainer est surtout séduite par la volonté affichée par Robbe-Grillet de renouveler son regard sur le monde en œuvrant à la construction d'un réalisme débarrassé des allégories métaphysiques et du romantisme. Ainsi, elle fait sienne cette déclaration du théoricien du nouveau roman :

«La réalité ne serait plus sans cesse située ailleurs, mais ici et maintenant, sans ambiguïté. Le monde ne trouverait plus sa justification dans un sens caché, quel qu'il soit, son existence ne résiderait plus que dans sa présence concrète, solide, matérielle ; au-delà de ce que nous voyons (de ce que nous percevons par nos sens) il n'y aurait désormais plus rien ${ }^{14}$.»

Cette citation est un parfait condensé de ce que Rainer a puisé chez l'écrivain. Effectivement, Robbe-Grillet refuse de s'approprier le monde et souhaite décrire les objets en surface sans leur présupposer une profondeur, une existence au-delà de leur phénomène. " C'est avant tout dans sa présence que réside [la] réalité [du monde] ${ }^{15}$ », dit-il. En définitive, RobbeGrillet donne le privilège narratif aux objets, créant ainsi ce que Barthes a appelé une littérature « objective » ou « littérale » ${ }^{16}$. L'écrivain s'attache à décrire, de manière extrêmement minutieuse et détaillée, des objets en apparence anodins ou insignifiants. Ôtant à ces derniers toute fonction et toute substance, il se concentre sur leurs qualités matérielles objectives. Il s'intéresse aux formes, aux matières, aux couleurs, mais ne fait que les observer, poser sur eux le regard le plus indifférent qui soit. En ce sens, si sa littérature est objective c'est - comme Barthes l'a démontré dans son analyse critique des premiers romans de Robbe-Grillet (Les gommes et Le voyeur) - au sens optique du terme. Délaissant la description classique qui donne la part belle au tactile, donc au viscéral, Robbe-Grillet offre la primauté au visuel. C'est le regard sur le monde extérieur qu'il se propose de réformer afin de rompre avec l'objet classique métaphorique. Selon l'écrivain, il faut regarder le monde tel « un homme qui marche dans la ville sans d'autre horizon que le spectacle, sans d'autre pouvoir que celui-là même de ses yeux ${ }^{17}$ ». On retrouve là la démarche de Rainer, dont les performeurs ne sont jamais rien d'autre qu'eux-mêmes, n'interprètent rien. Elle confronte ces derniers à un monde d'objets qui, loin des traditionnels accessoires au rôle dramaturgique ou esthétique, ne renvoie qu'au réel le plus trivial. Ce qu'elle donne à voir, c'est le quotidien qui se déploie hors de la scène mais repensé de manière à attirer l'attention sur certains gestes et certaines formes. De même, la chorégraphe impose aux danseurs ce regard indifférent sur leur entour. Par le biais de cette neutralité, elle saisit l'opportunité de se concentrer sur l'essentiel, à savoir le mouvement lui-même. Comme chez Robbe-Grillet, cela passe par la description et la répétition. L'exhaustivité dont l'écrivain fait preuve ainsi que sa tendance à ressasser les mêmes descriptions de manière quasiment litanique confèrent aux objets une présence entêtante, presque obsessionnelle. De son côté, Rainer propose une danse particulièrement descriptive et itérative. On pense notamment à l'enregistrement sonore qui accompagne PoSS : ces chroniques consciencieusement et excessivement détaillées des événements de la vie quotidienne au XIX ${ }^{\mathrm{e}}$

\footnotetext{
13 Yvonne Rainer, Feelings are Facts, Cambridge Mass., MIT Press, 2006. p. 397

${ }^{14}$ Alain Robbe-Grillet, Pour un nouveau roman, Paris, Gallimard, 1963, p. 44.

15 Ibid, p. 25.

16 Roland Barthes, «Littérature objective », in Essais Critiques, Paris, Edition du Seuil,1964.

${ }^{17}$ Roland Barthes, « Littérature objective », art. cit., p.39.
} 
siècle à Salem, petite ville du Massachussetts. Rainer a ici recours à l'un de ses procédés favoris, la juxtaposition radicale, consistant à confronter une bande sonore et des mouvements qui n'entretiennent aucun rapport a priori, mais qui, par le biais du collage, produisent du sens de manière inattendue. Dans PoSS l'obsession du détail banal présente dans le texte du Révérend Bentley résonne dans la danse elle-même, où les mêmes séquences de mouvements sont inlassablement répétées afin, justement, de permettre aux spectateurs de les détailler parfaitement. L'insistance sur la répétition constante des éléments les plus insignifiants engendre une temporalité sans finalité, où la danse semble n'avoir aucun but, et accuse autant de difficultés à évoluer que le pesant matelas. On le voit, la chorégraphie même est calquée sur l'objet, dont elle reproduit les propriétés physiques - lourdeur, lenteur, consistance, insignifiance. De même, Robbe-Grillet répète et varie une cellule narrative première, jamais conduite à son terme, mais qui revient sans cesse aux mêmes objets, sans jamais présenter autre chose que leur surface.

\section{Non à l'anthropomorphisation du monde}

Dans le roman traditionnel, les choses sont généralement investies d'une plus-value, elles sont porteuses de significations et sont le récipient des sentiments humains. Comme le résume Barthes:

«Le réalisme traditionnel additionne des qualités en fonction d'un jugement implicite : ses objets ont des formes, mais aussi des odeurs, des propriétés tactiles, des souvenirs, des analogies, bref ils fourmillent de significations; ils ont mille modes d'être perçus, et jamais impunément, puisqu'ils entraînent un mouvement humain de dégoût ou d'appétit ${ }^{18 》 \text { ». }}$

Analysant l'art du roman, Robbe-Grillet dénonce ce qu'il appelle la tromperie humaniste, qui contamine le texte dans son ensemble en présentant un " univers tragifié ». Ce faisant, le roman traditionnel vise à sublimer la distance entre l'homme et le monde en l'élevant au rang de suprême souffrance. Robbe-Grillet cite l'exemple de La Nausée de Sartre où l'univers entier, vu à travers les yeux de Roquentin, est soumis au triste dégoût ressenti par le héros. Celui-ci déclare : « tous les objets qui m'entouraient étaient faits de la même matière que moi, d'une espèce de souffrance moche ${ }^{19}$ ». Pour Robbe-Grillet, « dans cet univers peuplé de choses, celles-ci ne sont plus pour l'homme que des miroirs qui lui renvoient sans fin sa propre image ${ }^{20}$ ». C'est en réaction contre ce qu'il dénonce comme une supercherie humaniste qui revient à affirmer que « le monde, c'est l'homme », que l'écrivain se concentre sur la description la plus neutre possible des choses. Cet antihumanisme qui conduit à une redéfinition de l'objet est l'une des marques de fabrique des artistes commodément rassemblés sous la bannière de l'art minimal, mouvement auquel les critiques ont souvent affilié Rainer ${ }^{21}$. Carl Andre, Donald Judd, Dan Flavin ou Robert Morris rejettent eux aussi l'illusionnisme et l'humanisme anthropocentrique tout comme la relation subjective et affective aux œuvres d'art ${ }^{22}$. En mettant l'accent sur les qualités matérielles des objets qu'ils créent, sur leur présence physique, ils engendrent une relation purement phénoménale avec l'œuvre. Cependant, la remise en question de l'anthropomorphisation du monde semble beaucoup plus difficilement réalisable en danse.

De fait, en tant qu'art de mouvoir le corps humain de manière à configurer l'espace et le temps, la danse a traditionnellement été perçue comme le médium privilégié de l'expression des

18 Roland Barthes, « Littérature objective », art. cit., p.30.

19 Jean-Paul Sartre, La Nausée, Paris, Gallimard, 2005, p. 244.

20 Alain Robbe-Grillet, Pour un nouveau roman, op. cit., p.77.

21 Voir entre autres Barbara Rose, «ABC Art », art. cit.; Robin Silver Hecht, «Reflections on the Career of Yvonne Rainer and the Values of Minimal Dance », Dance Scope, Automne-hiver 1973/74, vol. 8, n¹, pp. 5-6. Il faut ajouter à cela que l'œuvre de Rainer entretient de nombreuses affinités avec l'œuvre de Robert Morris puisque les deux artistes ont travaillé sur plusieurs chorégraphies ensemble et ont vécu une relation amoureuse plutôt tumultueuse.

22 Voir par exemple Frances Colpitt, Minimal Art. The Critical Perspective, Seattle, University of Washington Press, 1990. 
sentiments et des passions humaines. Si l'on prend l'exemple de la danse moderne particulièrement significatif car Rainer s'est ostensiblement affranchie de ce mouvement et en a pris le contrepied - l'intensité du sentiment détermine l'intensité du geste et on attribue à chaque sentiment une traduction corporelle propre. Dans ce contexte, l'objet présenté sur scène n'est jamais utilisé pour lui-même, en raison de ses caractéristiques spécifiques, mais est généralement choisi en vertu de sa dimension métaphorique faisant écho aux affects des danseurs. Ceci est manifeste dans la danse de Martha Graham, tournée vers l'introspection psychologique et focalisée sur l'être humain et ses conflits. À mille lieux des objets ordinaires, inexpressifs et impénétrables, les choses sont employées comme faire-valoir des protagonistes et sont surchargées de signification. Citons, par exemple, le ballet Frontier (1935) de Martha Graham. Le décor de ce solo, très sobre, a été conçu par Isamu Noguchi et se compose de deux cordes et d'un banc de bois, qui illustrent le thème de la chorégraphie : l'esprit de la culture américaine. Ainsi, le dénuement même de l'espace suggère l'immensité du paysage américain ainsi que la soif de liberté. Le banc, lui, symbolise la frontière et les deux cordes font allusion aux rails du chemin de fer traversant les États-Unis. On le voit, les objets ont ici pour mission de mettre en exergue les émotions de la pionnière américaine. C'est là une utilisation métaphorique de la chose dont Rainer souhaite s'éloigner. De même, elle refuse que l'objet soit réduit au rôle de faire-valoir des danseurs. C'est pourquoi elle se montre parfois tout aussi critique à l'encontre de la danse postmoderne :

«Les objets ont [jusque là] été utilisés hors des limites de leur présence immédiate,

comme des intermédiaires pour créer des événements et, invariablement, dans ces événements, le centre de l'attention a été les performeurs humains, et ce aussi minimale que puisse être leur performance ${ }^{23}$.»

De fait, dans PoSS, les matelas détiennent un rôle déterminant : les danseurs les manipulent, les transportent, s'y couchent ou sautent dessus, s'enroulent dedans, rampent en dessous, etc. En se débarrassant de la suprématie habituellement conférée à la danseuse ou au danseur, Rainer met en échec l'anthropocentrisme, qui se manifeste selon elle à travers le narcissisme et le culte de la personnalité, qu'elle fustige ardemment dans son NO Manifesto ${ }^{24}$. Ainsi, elle conçoit l'objet comme un excellent rempart contre toute relation narcissique entre les danseurs et les spectateurs. En effet, en offrant une place décisive à l'objet dans la chorégraphie, elle dirige l'entière attention des performeurs vers celui-ci, les empêchant ainsi d'être polarisés sur leurs propres corps ${ }^{25}$. L'intériorité étant mise entre parenthèses, la danse se concentre alors sur une expérience directe, purement spatiale et temporelle, de la réalité. Il faut d'ailleurs noter que le sous-titre de la pièce (" une danse pour dix personnes et douze matelas ») instaure une relation d'égalité entre danseurs et objets. Les matelas sont conçus comme des performeurs à part entière, en dépit de leur inertie et de leur non-humanité. Mais, réciproquement, les corps humains sont eux aussi maniés, ramassés, transportés, et ainsi chosifiés. Clamant qu'idéalement le performeur «ne doit pas même être lui-même, il doit être un exécutant neutre ${ }^{26}$ », Rainer va jusqu'à affirmer que « les objets et les corps peuvent être interchangeables ${ }^{27}$ », remettant de ce fait totalement en cause la hiérarchie entre l'animé et l'inanimé. Confrontant les propriétés physiques de chacun, la chorégraphe met ainsi en place une relation complémentaire très inhabituelle en danse. Sa conception du corps-objet aboutit à un dépassement du traditionnel clivage objet-sujet.

23 Yvonne Rainer, "Notes on Two Dances by Deborah Hay", Ikon, vol.1, n¹, p.2.

${ }^{24}$ Yvonne Rainer s'est fréquemment exprimée sur ce qu'elle appelle la «nature exhibitionniste » de la danse. « En tant que danseuse j'avais fortement conscience d'être un "objet de regard" » dit-elle et de solliciter sans cesse une « confrontation immédiate avec le regard aimant du spectateur» (Liza Bear, Willoughby Sharp, " The Performer as Persona: An Interview with Yvonne Rainer », Art. Cit. p. 52).

25 Ibidem.

26 Yvonne Rainer, "A Quasi Survey of Some 'Minimalist' Tendencies in the Quantitatively Minimal Dance Activity Midst the Plethora, or an Analysis of Trio A", in Work 1961-1973, Halifax, The Press of Novia Scotia College of Art and Design, 1974, p. 65.

${ }^{27}$ Liza Bear, Willoughby Sharp, « The Performer as Persona: An Interview with Yvonne Rainer », Avalanche, $\mathrm{n}^{\circ}$ 5, été 1972, p. 50. 
Inévitablement, cette déhiérarchisation transforme l'art chorégraphique en profondeur, interrogeant notamment le rapport au public.

Durant les années soixante, la phénoménologie a été une source d'inspiration importante pour les auteurs du nouveau roman comme pour les artistes gravitant autour du minimalisme ${ }^{28}$. Ces derniers se sentent stimulés par le primat que la pensée phénoménologique donne au subjectif et au phénomène et par sa volonté d'éliminer tout a priori dans la relation que l'individu entretient avec le monde. En définitive, la théorie du nouveau roman développée par Robbe-Grillet reprend très précisément les termes de Merleau-Ponty, selon lequel «le monde est là avant toute analyse que je puisse en faire et il serait artificiel de le faire dériver d'une série de synthèses ${ }^{29}$. Influencée par la phénoménologie de la perception, la danse de Rainer s'efforce de provoquer une expérience perceptive et cognitive chez la spectatrice ou le spectateur : elle propose une danse réflexive qui encourage le public à adopter une position de sujet conscient et critique. Surtout, elle s'intéresse au phénomène en tant que la chose elle-même qui se montre. De fait, dans la danse de Rainer les objets sont là, tout simplement, chacun limité à soi. En se positionnant "en face» d'eux, la chorégraphe s'efforce de donner à l'objet un "être là" et de lui ôter un "être quelque chose"30. On retrouve là les termes de la pensée heideggérienne, qui montre que le corps pensant doit se comprendre lui-même à partir de ce qui n'est pas lui, et non plus comprendre le monde à partir de lui. Ainsi, selon Heidegger, c'est à travers la compréhension de l'être de l'objet que l'individu a accès à l'être même du monde ${ }^{31}$. En régénérant le geste dansé à travers une nouvelle attitude globale de présence au monde, totalement engagée dans la quotidienneté et l'ordinaire, Rainer s'efforce d'atteindre cet être-dans-le-monde. Car la manière d'être dans le monde de l'individu est en premier lieu pratique, nous dit Heidegger, c'est ainsi que le monde se découvre à lui. Aussi, en proposant une expérience de la banalité dans sa danse, la chorégraphe souligne qu'être humain c'est être immergé dans le monde le plus concret et quotidien. Dès lors, Rainer se débarrasse totalement de la notion de spectacle et offre au public la possibilité d'expérimenter une relation véritablement substantielle avec le réel.

On l'a vu, il est particulièrement éclairant de mettre en regard le rôle de l'objet dans la danse de Rainer et la conception du monde matériel développée par Robbe-Grillet. Moment charnière dans la carrière de l'artiste, PoSS engendre une réflexion sur la nature même de la danse qui découle directement de la redéfinition de l'objet comme un sujet et du sujet comme un objet. À partir de cela, Rainer parvient à formuler et à théoriser sa révolte contre les codes établis de la danse. Il semble que la reconsidération de l'objet lui ait permis de laisser émerger ses aspirations profondes et de faire évoluer le mouvement en le modelant sur l'être-là de l'objet. Néanmoins, en conclusion, il est intéressant d'apporter quelques nuances à notre réflexion. En effet, de son propre aveu jamais aussi radicale et minimaliste que son collègue du Judson Dance Theater, Steve Paxton, Rainer n'abandonna en définitive guère complètement le "glamour loufoque » de ses débuts. Aussi, en ne mettant en évidence que ses aspects les plus dépouillés, austères et minimalistes, on oublie trop souvent que sa danse n'éludait pas complètement les aspects métaphoriques. Ainsi dans PoSS, la chorégraphe ne renie pas la dimension sexuelle de ce fameux matelas, bien au contraire. Durant la phase de conception de cette chorégraphie, elle avait élaboré

28 Voir entre autres, Rosalind E. Krauss, Passages in Modern Sculpture, Cambridge Mass., The MIT Press, 1977; Victor Carrabino, "Phenomenology and the 'Nouveau Roman': A Moment of Epiphany", South Atlantic Bulletin, vol. 38, $\mathrm{n}^{\circ} 4$, novembre 1973, pp. 95-100. L'influence de la phénoménologie est particulièrement évidente dans le travail de Robert Morris, qui propose une lecture phénoménologique de ses «formes unitaires », centrée sur l'expérience de l'œuvre par le spectateur, dans son article « Notes on sculpture», Artforum, vol. 4, n6, février 1966, pp. 42-44. Or, comme on l'a précisé précédemment, Rainer a entretenu un dialogue fécond avec cet artiste durant une bonne partie des années 1960.

${ }^{29}$ Maurice Merleau-Ponty, Phénoménologie de la perception, Paris, Gallimard, 1945, p. iv.

30 On reprend ici les propos de Roland Barthes à propos du nouveau roman de Robbe-Grillet. Voir Roland Barthes, « Littérature objective », art. cit., p. 31.

31 Martin Heidegger, Etre et Temps, Paris, Gallimard, 1986. 
un duo explicitement érotique avec Robert Morris intitulé Part of a Sextet (fragment d'un sextuor). Il est loin d'être anodin qu'elle ait conservé ce jeu de mot volontairement ringard et grivois dans le titre de la pièce finale, à la fois comme une marque du sens de l'humour omniprésent dans son œuvre et en guise de pied de nez à la doxa minimaliste, dans laquelle elle ne se reconnaissait pas entièrement. Car il ne faut pas oublier une dimension essentielle du travail de Rainer : il s'agit d'un art de la juxtaposition et de la disjonction qui conserve toujours un caractère ironique. Aussi, il ne lui apparaît pas contradictoire de souscrire au rejet de la métaphore prôné par le nouveau roman tout en glissant une allusion au sens caché que peut revêtir l'objet. Ce faisant, elle va jusqu'au bout d'une démarche qui vise à offrir aux spectateurs une totale liberté en n'interdisant aucune clé d'interprétation.

\section{Bibliographie}

BARTHES, Roland, «Littérature objective», Critique, vol. X, n 86-87, juillet-août 1954, p. 581-591.

HEIDEGGER, Martin, Etre et Temps, Paris, Gallimard, 1986.

RAINER, Yvonne, "Some Retrospective Notes on a Dance for 10 People and 12 Mattresses called "Parts of Some Sextets," Performed at the Wadsworth Athenaeum, Hartford, Connecticut, and Judson Memorial Church, New York, in march, 1965 », TDR, vol. 10, n² 2, winter 1965, pp. 168178.

RAINER, Yvonne, "A Quasi Survey of Some 'Minimalist' Tendencies in the Quantitatively Minimal Dance Activity Amidst the Plethora, or an Analysis of Trio A », in Gregory Battcock (ed.), Minimal Art, a Critical Anthology, [1968], Berkeley, University of California Press, 1995, pp. 263273.

RAINER, Yvonne, A Woman Who... Essays, Interviews, Scripts, Baltimore, John Hopkins University Press, 1999.

RAINER, Yvonne, Feelings are Facts, a Life, Cambridge Mass., MIT Press, 2006.

RAINER, Yvonne, Work 1961-1973, Halifax, The Press of Novia Scotia College of Art and Design, 1974.

ROBBE-GRILLET, Alain, Pour un nouveau roman, Paris, Gallimard, 1963.

\section{Pour citer ce document}

Johanna Renard, « Un arrangement d'objets et de personnes : étude de Parts of Some Sextets d’Yvonne Rainer, Agôn [en ligne], Dossiers, $N^{\circ} 4$, L’Objet, L'objet, le corps : de la symbiose à la confrontation, mis à jour le : 22/12/2011, URL : http://agon.ens-lyon.fr/index.php?id=1988 